\title{
Comparison of different promoter methylation assays in breast cancer
}

\author{
Karijn P.M. Suijkerbuijk ${ }^{\mathrm{a}}$, Xiaojuan Pan ${ }^{\mathrm{a}}$, Elsken van der Wall ${ }^{\mathrm{c}}$, Paul J. van Diest ${ }^{\mathrm{a}, *}$ and Marc Vooijs ${ }^{\mathrm{a}, \mathrm{c}}$ \\ ${ }^{a}$ Department of Pathology, University Medical Center Utrecht Cancer Center, Utrecht, The Netherlands \\ ${ }^{\mathrm{b}}$ Division of Internal Medicine and Dermatology, University Medical Center Utrecht Cancer Center, Utrecht, \\ The Netherlands \\ ${ }^{\mathrm{c}}$ Department of Radiation Oncology (Maastro Lab), GROW School for Oncology and Developmental Biology, \\ Maastricht University, Maastricht, The Netherlands
}

\begin{abstract}
Background: Promoter hypermethylation has emerged as a promising cancer biomarker. Currently, a large variety of quantitative and non-quantitative techniques is used to measure methylation in clinical specimens. Here we directly compared three commonly used methylation assays and assessed the influence of tissue fixation, target sequence location and the amount of DNA on their performance.

Methods: We used Methylation-Specific PCR (MSP), Quantitative Multiplex MSP (QM-MSP) and Methylation-Specific Multiplex Ligation-dependent Probe Amplification (MS-MLPA) to compare methylation of CCND2, SCGB3A1, RARB and RASSF1 on DNA from 40 breast carcinomas.

Results: A comparison between MSP and QM-MSP on the same samples showed a high discrepancy: 20\% of tumors that showed no methylation in MSP gave >10\% methylation in QM-MSP. In contrast, QM-MSP correlated strongly with MS-MLPA when targeting the same sequence in DNA from paraffin embedded as well as fresh frozen tissue. This correlation declined when target sequences were non-overlapping. In titration experiments, MSP and MS-MLPA performed robust with 10 ng of DNA, while QM-MSP was at least ten-fold more sensitive.

Conclusion: Despite the difference in molecular basis, QM-MSP and MS-MLPA showed moderate to strong correlations. In contrast, there was a poor concordance between either of these techniques and non-quantitative MSP. For biological samples with scarce DNA, QM-MSP is the method of choice.
\end{abstract}

Keywords: Breast cancer, DNA methylation, methodology, MS-MLPA, MSP, QM-MSP

\section{Introduction}

Over the last decade, the importance of epigenetic silencing of tumor suppressor genes by DNA promoter hypermethylation has been recognized as a common mechanism driving carcinogenesis [10]. Methylation has proven to be a promising predictive and prognostic biomarker [19], but is especially promising as a biomarker for early cancer detection in biological fluids [21] for various reasons. First, methylation aberrations occur in high frequency and in early stages of tumor development $[14,18]$. Secondly, methylation changes

\footnotetext{
* Corresponding author: Prof. Paul J. van Diest, MD, PhD, Department of Pathology, University Medical Center Utrecht Cancer Center, PO Box 85500, 3508 GA Utrecht, The Netherlands. Tel.: +31 88 7556565; Fax: +31 30 2544990; E-mail: P.J.vanDiest@ umcutrecht.nl.
}

have been demonstrated in non-cancerous cells adjacent to the tumor [26]. This so-called field defect facilitates detection in heterogeneous biological samples. Lastly, methylation detection is applicable to biological fluids that contain low amounts of DNA [3,7,22].

This has in recent years lead to the development of a large variety of Polymerase Chain Reaction (PCR) based methylation assays interrogating methylation of cytosine (methyl-C) in $\mathrm{CpG}$ dinucleotides that cluster in $\mathrm{CpG}$ islands in the regulatory sequences of gene-promoters [13]. Detection of methyl-cytosines is based on either sodium bisulfite (NaBi) conversion of unmethylated cytosine to uracil [6] or on the use of methylation-sensitive DNA restriction enzymes [8]. The most direct and comprehensive way to provide information on methylation is genomic sequencing of $\mathrm{NaBi}$ treated DNA, however this is laborious and cumbersome when methylation is only 
present in a subset of cells within a clinical specimen. Methylation-Specific PCR (MSP) is the most simple, affordable and commonly used $\mathrm{NaBi}$ treatment based detection method for gene methylation in patient material, but is non-quantitative. In contrast, quantitative PCR based methods, such as Quantitative Multiplex Methylation-Specific PCR (QM-MSP) [4] enable the detection of methylated genes at very high sensitivity (1:10,000 cells) in complex samples. A powerful alternative approach is the semi-quantitative MethylationSpecific Multiplex Ligation-dependent Probe Amplification (MS-MLPA) that relies on methylation-sensitive endonucleases that discriminate between methylated and unmethylated DNA sequences [17]. Like MLPA [16], MS-MLPA is relatively fast and inexpensive. The assays compared here are representatives of the large spectrum of $\mathrm{NaBi}$ and restriction enzyme based PCR based methylation assays that is still expanding [12].

Although methylation analysis through the use of methylation-specific restriction endonucleases is fast and simple, fixation may influence the efficiency of enzyme cleavage [13] and lead to false positive results [15], an important consideration when analyzing archival patient material. Furthermore, in these assays methylation analysis is restricted to methylation-sensitive restriction sites. Since methylation of $\mathrm{CpG}$ dinucleotides may vary within one $\mathrm{CpG}$ island [1], the choice of different primer sets between different studies makes extrapolation of these findings cumbersome. Finally, for methylation analysis in biological samples with low DNA content, such as nipple aspirate fluid [22] and plasma [7], sensitivity is of the utmost importance.

Obviously, it is important to know how well different kinds of methylation assays compare, in order to be able to extrapolate information on methylated genes from one study to another. Although this issue does not seem to be of minor importance, to date, no direct comparison has been reported of quantitative and non-quantitative $\mathrm{NaBi}$ and restriction enzyme methylation assays. Here we compared MSP, QM-MSP and MS-MLPA side by side and investigated the influence of tissue fixation, primer location and amount of DNA on methylation detection of RARB, RASSF1, CCND2 and $S C G B 3 A 1$; four genes previously reported to be frequently methylated in breast cancer $[4,20]$.

\section{Methods}

\subsection{Tissue selection and DNA isolation}

Forty invasive breast carcinomas, selected based on the availability of formalin fixed paraffin embedded as well as fresh frozen specimens were obtained from the Pathology department of the University Medical Center Utrecht. Use of anonymous or coded left-over material for scientific purposes is part of the standard treatment contract with patients in our hospital [24]. H\&E-stained slides of the paraffin blocks were reviewed by a pathologist (PvD) to confirm the presence of malignancy. The percentage of carcinoma cells in each tissue section was estimated to be at least $50 \%$. DNA from formalin fixed paraffin embedded and fresh frozen tissue was isolated as described previously [5].

\section{2. $M S P$}

MSP was performed according to Herman et al. [6]. Sodium bisulfite $(\mathrm{NaBi})$ treatment was performed using the Epitect Kit (Qiagen). The PCR mixture contained $1 \times$ MSP buffer $\left(16.6 \mathrm{mM} \mathrm{NH}_{4} \mathrm{SO}_{4}, 67 \mathrm{mM}\right.$ Tris $\mathrm{pH} 8.8,6.7 \mathrm{mM} \mathrm{MgCl}_{2}, 10 \mathrm{mM} \beta$-mercaptoethanol, $0.1 \%$ DMSO), $1.25 \mathrm{mM}$ dNTP, primers (300 ng each), 1.25 U Platinum Taq (Invitrogen) and approximately $100 \mathrm{ng}$ of bisulfite-modified DNA. Reactions were hotstarted at $95^{\circ} \mathrm{C}$ for $5 \mathrm{~min}$, followed by 35 amplification cycles $\left(30 \mathrm{~s}\right.$ at $95^{\circ} \mathrm{C}, 30 \mathrm{~s}$ at $56^{\circ} \mathrm{C}$ and $30 \mathrm{~s}$ at $72^{\circ} \mathrm{C}$ ) and a 4 min extension at $72^{\circ} \mathrm{C}$. Human sperm DNA was used as a negative control and Sss I methylase treated Human Sperm DNA as a positive control. Controls without DNA were included in each PCR. $10 \mu \mathrm{l}$ of each PCR product was loaded onto a $2 \%$ agarose gel containing ethidium bromide, and visualized by UV illumination.

\section{3. $Q M-M S P$}

QM-MSP was performed as described previously [4,20,23]. After conventional NaBi treatment, multiplex PCR (35 cycles) was performed using external primers independent of DNA methylation status to coamplify $R A R B, R A S S F 1, C C N D 2$ and $S C G B 3 A 1$ using previously published primers $[3,4]$. In the quantitative step, a two-color real-time MSP ( 40 cycles) was done with internal primers and 6-FAM/TAMRA and VIC/TAMRA probes as described [3,4], targeting the same sequences as the MSP primers. A standard curve and a $80 \mathrm{~K}$ copy number control were used for calculating percentage methylation $([\mathrm{M} /(\mathrm{U}+\mathrm{M})] \times 100)$.

\subsection{Methylation-specific MLPA (MS-MLPA)}

The principles of MLPA and MS-MLPA have been described elsewhere [17]. MS-MLPA probes contain 
Table 1

Genes used to compare different quantitative methylation assays and comparison of their target sequences $($ par $=$ paraffin, $\mathrm{ff}=$ fresh frozen $)$

\begin{tabular}{|c|c|c|c|c|c|}
\hline Gene & $\begin{array}{c}\text { Number of CpG } \\
\text { nucleotides } \\
\text { analyzed by } \\
\text { QM-MSP }\end{array}$ & $\begin{array}{l}\text { MS-MLPA probe } \\
\text { name }\end{array}$ & $\begin{array}{l}\text { MS-MLPA probe } \\
\text { sequence overlaps } \\
\text { with QM-MSP } \\
\text { target sequence }\end{array}$ & $\begin{array}{c}\text { Distance in base pairs } \\
\text { between MS-MLPA } \\
\text { and QM-MSP target } \\
\text { sequences }\end{array}$ & $\begin{array}{c}\text { Number of CpG } \\
\text { nucleotides } \\
\text { analyzed by } \\
\text { MS-MLPA }\end{array}$ \\
\hline \multirow[t]{3}{*}{$C C N D 2$} & 3 & 03313-L02668 & Yes & - & 1 \\
\hline & & 03312-L09381 (par) & No & 129 & 1 \\
\hline & & 03312-L2667 (ff) & & 127 & \\
\hline \multirow[t]{3}{*}{$S C G B 3 A 1$} & 3 & 03305-L09382 (par) & Yes & - & 1 \\
\hline & & 03305-L2657 (ff) & & & \\
\hline & & 11132-L12956 & No & 56 & 2 \\
\hline$R A R B$ & 2 & 4040-L01698 & No & 508 & 1 \\
\hline \multirow[t]{2}{*}{$R A S S F 1$} & 4 & 3807-L02159 & Yes & - & 1 \\
\hline & & 2248-L01734 & No & 32 & 1 \\
\hline
\end{tabular}

a recognition sequence for the methylation-sensitive restriction enzyme HhaI. We used the SALSA MSMLPA ME001-C1 and ME003-A1 kit (MRC-Holland, Amsterdam, The Netherlands) that contain 26, respectively 27 probes targeted against the promoters of a set of genes that are frequently silenced by methylation in various tumors. Besides from the HhaI-sensitive targets, 15 respectively 13 different control probes were present that were not influenced by the HhaI digestion. These two kits contain probes that partly do and partly do not overlap the QM-MSP target sequences for RASSF1, SCGB3A1 and CCND2, which enabled us to evaluate the influence of probe localization on methylation results. For $R A R B$, only a non-overlapping probe was available. In Table 1 the positions of the MS-MLPA-probes relative to the QM-MSP target sequences are given. We did not analyze methylation data for the other methylation targets present in the two MSMLPA kits, since these data are not relevant for the comparative goal of this study. After $16 \mathrm{~h}$ of hybridization of the probes to the sample DNA, the sample reaction was divided into two parts. The first part was only ligated and provides information on copy number changes. The second part of the MS-MLPA hybridization reaction was digested with HhaI while the hybridized probes were ligated. Hybrids of probe DNA and unmethylated sample DNA were digested by the HhaI enzyme and thus could not be amplified by PCR (35 cycles), while the methylated DNA could not be digested and the ligated probes generated a probe signal after PCR. Each MS-MLPA reaction of a DNA sample then yielded two results to be analyzed; one undigested sample that was only ligated (total DNA) and one sample that was simultaneously digested and lig- ated (methylated DNA). Samples were run on a 310 DNA Analyzer (Applied Biosystems). The ratio of the two reactions was converted to a percentage methylation, using the Coffalyser software [2].

\subsection{Dilution experiment}

To define the range of DNA concentrations in which the different assays can detect methylation, we used serial DNA dilutions from the human breast epithelial MCF10A cell line of 200, 100, 50, 25, 10, $1 \mathrm{ng}$ and $100 \mathrm{pg}$, respectively. With these decreasing amounts of input DNA (before NaBi or restriction enzyme treatment), we performed MS-MLPA, QM-MSP and MSP for $R A R B$ as described above, with the only difference that for this comparison both QM-MSP and MSP were performed using conventional NaBi modification (as described in the QM-MSP section).

\subsection{Statistics}

Results for the four separate genes were pooled for analysis. Spearman correlations coefficients $\left(r_{\mathrm{s}}\right)$ were calculated. Curve estimations and scatterplots were used to depict the associations between different assays. A boxplot was used to look at the concordance between QM-MSP and MSP. SPSS 15.0 for Windows was used for statistical analysis.

\section{Results}

\subsection{Poor concordance between quantitative and non-quantitative assays}

As methylation analysis is becoming increasingly important in clinical decision making we sought to 
compare representatives of the most commonly used methylation analysis techniques. To do so we compared DNA methylation frequencies of 4 commonly methylated genes in DNA isolated from 40 paraffinembedded invasive human breast cancer specimens measured by MSP, MS-MLPA and QM-MSP.

The non-quantitative assay MSP showed highly discrepant results when compared with the quantitative QM-MSP assay (Fig. 1). In 20\% of the samples that did not show a methylated product in MSP more than $10 \%$ methylation was found by QM-MSP. Moreover, in $10 \%$ of the samples that did not show methylation by MSP more than $50 \%$ methylation was observed by QM-MSP. Similar discrepancies were found when comparing MSP with MS-MLPA; $20 \%$ of the samples without a methylated product in MSP showed $30 \%$ methylation in MS-MLPA and in 10\% of the samples not displaying methylation in MSP even $100 \%$ methylation was found by MS-MLPA.

\subsection{Strong correlation between the quantitative assays QM-MSP and MS-MLPA}

When comparing both assays using overlapping target sequences and DNA extracted from paraffinembedded tissue, there was a strong correlation be- tween assays $\left(r_{\mathrm{s}}=0.67 ; p<0.001\right.$; Fig. 2a). When analyzing the genes individually, correlation coefficients were $0.65(p<0.001)$ for $C C N D 2,0.60$ $(p<0.001)$ for SCGB3A1 and $0.71(p<0.001)$ for RASSF1.

When comparing non-overlapping target sequences, the correlation decreased $\left(r_{\mathrm{s}}\right.$ for pooled analysis 0.43 ; $p<0.001$; Fig. 2b). The correlation between QMMSP and MS-MLPA with overlapping target sequences in fresh frozen tissue was comparable to the correlation in paraffin-embedded tissue $\left(r_{\mathrm{s}}=0.68\right.$; $p<0.001$; Fig. 2c). With non-overlapping sequences in fresh frozen tissue, the correlation was similar $r_{\mathrm{s}}$ of $0.64(p<0.001)$.

\subsection{Performance of $M S P, Q M-M S P$ and $M S-M L P A$ on small amounts of DNA}

Methylation analysis has potential to measure tumor-specific genomic alterations at high sensitivity as is necessary for the analysis of clinical specimens containing low amounts of DNA such as those harvested from biological fluids. Therefore we compared the detection range of the individual assays by making serial dilutions of MCF10A cell line DNA and determined the methylation frequencies in $R A R B$.

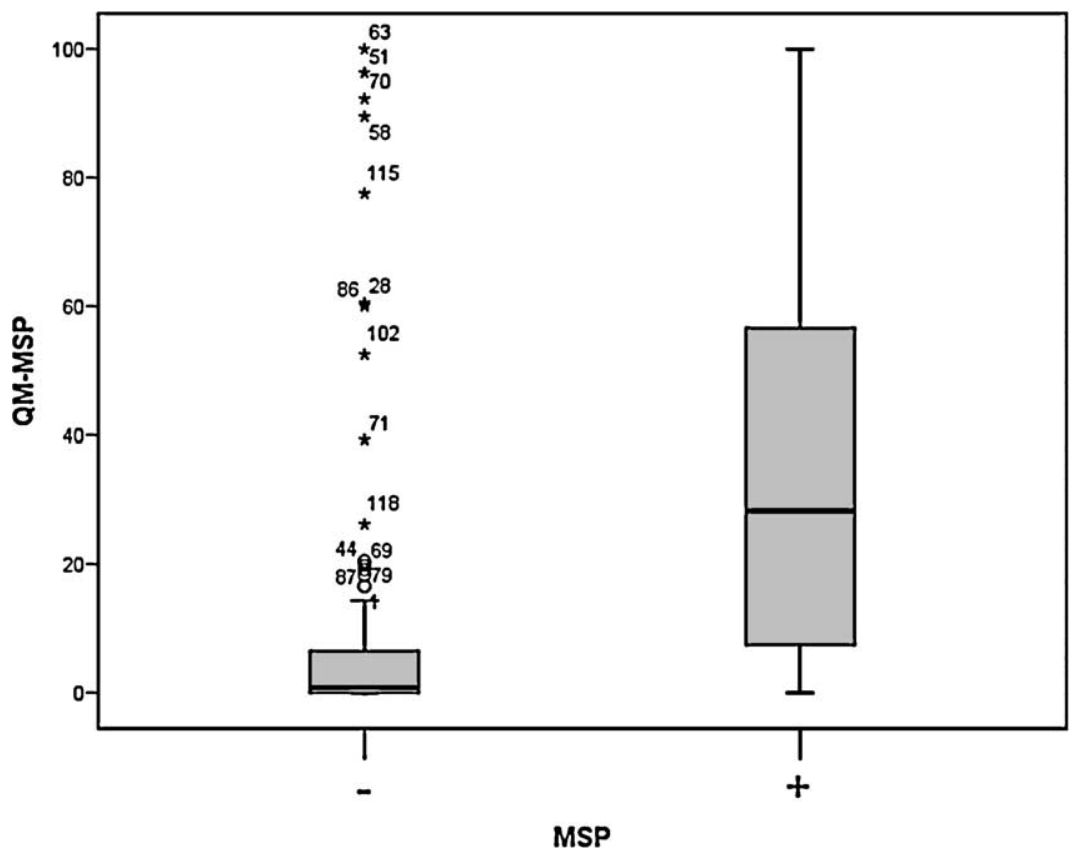

Fig. 1. Boxplot of methylation detection by QM-MSP versus MSP in breast cancer specimens $(n=40)$. The boxplot shows median $(-)$, interquartile range $(\mathrm{I})$ and outliers $\left(\mathrm{o}^{*}\right)$. The left plot shows the range of QM-MSP values (for DNA from paraffin-embedded tissue) for the samples that did not show a methylation band in MSP, the right panel shows the range for samples that did show a band in MSP. 


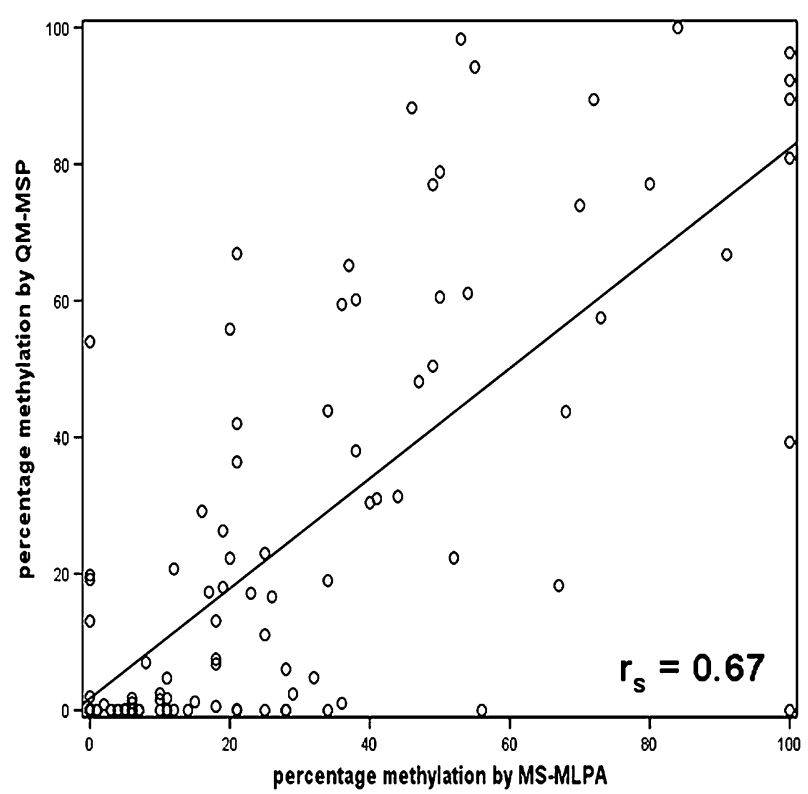

(a)

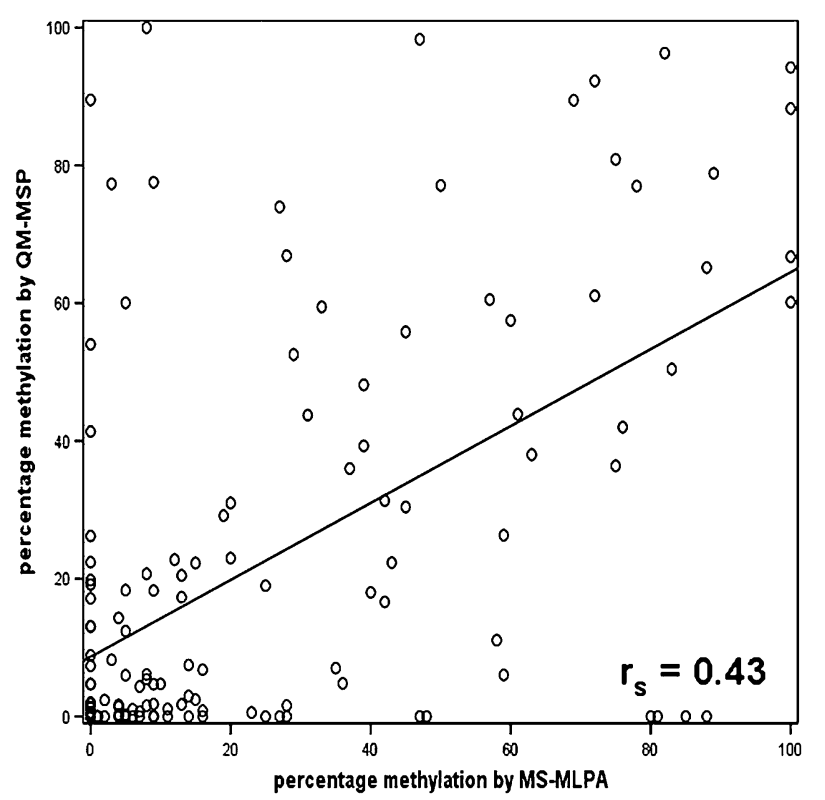

(b)

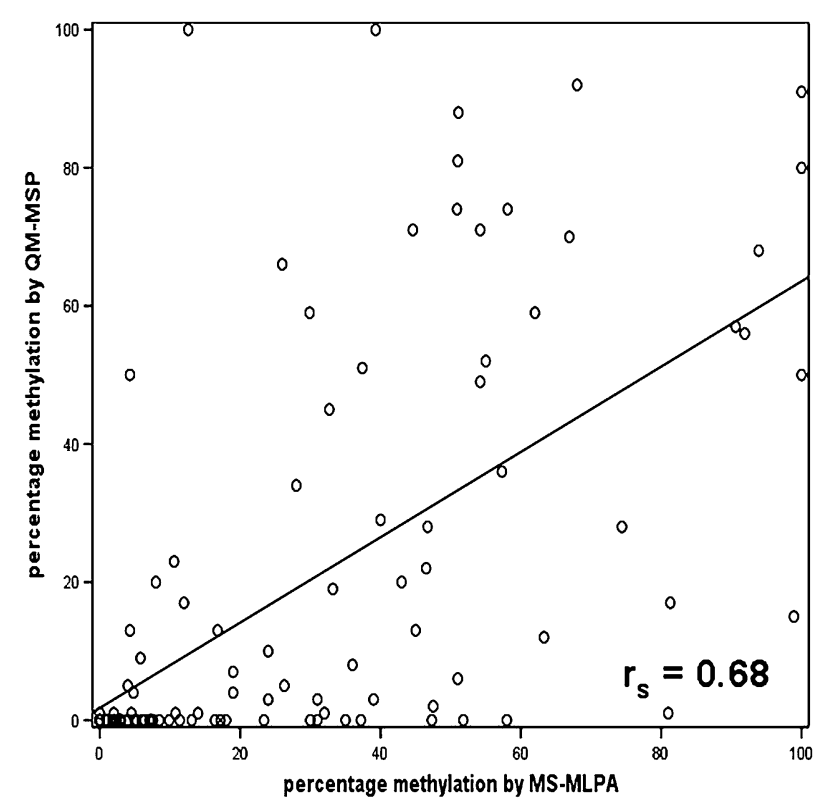

(c)

Fig. 2. Regression curve fitting to assess concordance of QM-MSP vs. MS-MLPA based methylation detection in breast cancer according to probe location and tissue fixation $\left(r_{\mathrm{s}}=\right.$ Spearman correlation coefficient): (a) MS-MLPA versus QM-MSP with overlapping target sequences using DNA from formalin fixed paraffin-embedded tissue; (b) MS-MLPA versus QM-MSP with non-overlapping target sequences using DNA from paraffin-embedded tissue; (c) MS-MLPA versus QM-MSP with overlapping target sequences using DNA from fresh frozen tissue.

As shown in Table 2 and Fig. 3, MS-MLPA and MSP still performed well in a dilution experiment when $10 \mathrm{ng}$ of input DNA was tested for $R A R B$ methylation. Note that the MSP only provides a qualitative output whereas QM-MSP provided distinct methylation frequencies. Importantly, QM-MSP still gave reproducible results with only $1 \mathrm{ng}$ of input DNA.

In all DNA concentrations, MSP for RARB showed $\mathrm{M}$ and $\mathrm{U}$ bands. In QM-MSP, methylation frequen- 
cies ranged from 76 to $83 \%$. In MS-MLPA methylation percentages were between 70 and $92 \%$, except for the $10 \mathrm{ng}$ sample, that showed $56 \%$ methylation, indicating the limit of the dynamic range for this assay.

Table 2

Results of a dilution to assess the minimum required input DNA for different non-quantitative (MSP) and quantitative (QM-MSP and MLPA) methylation assays ( + indicates positive signal for RARB)

\begin{tabular}{lcccc}
\hline Method & \multicolumn{4}{c}{ Total amount of input DNA (MCF10A) } \\
\cline { 2 - 5 } & $100 \mathrm{ng}$ & $10 \mathrm{ng}$ & $1 \mathrm{ng}$ & $100 \mathrm{pg}$ \\
\hline MSP & + & + & - & - \\
MS-MLPA & + & + & - & - \\
QM-MSP & + & + & + & - \\
\hline
\end{tabular}

\section{Discussion}

Here we compared methylation percentages in archival clinical breast cancer specimens for four genes using $\mathrm{NaBi}$ and restriction enzyme based methods. We observed a strong correlation between QM-MSP and MS-MLPA, when using overlapping probe sets with formalin fixed as well as with fresh frozen tissue samples. This indicates that neither enzyme nor NaBi treatment was affected significantly by tissue fixation in the 40 breast cancer specimens studied here. In contrast, when non-overlapping sequences were used, differences between assays were greater, highlighting the variability in $\mathrm{CpG}$ methylation between different regions of the same promoter. In a direct comparison
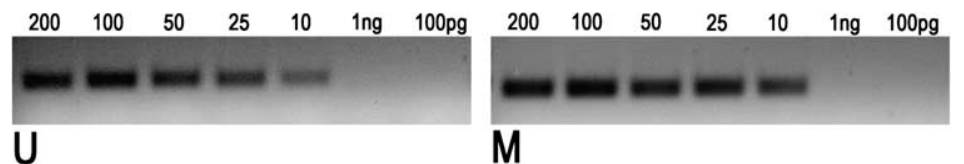

(a)
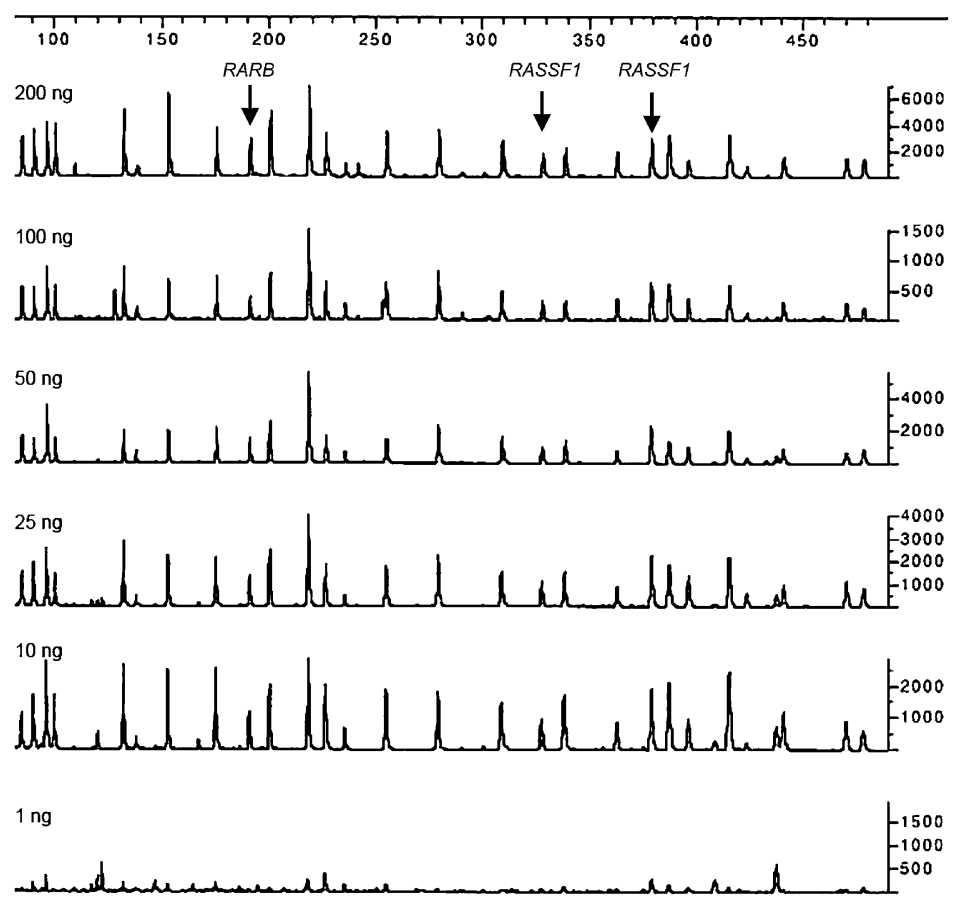

(b)

Fig. 3. Performance of different methylation assays in decreasing amounts of cell line DNA. (a) MSP for RARB on serial dilutions of MCF10A human breast epithelial cell line DNA. Amount of input DNA is indicated. MSP is feasible down to a minimum of $10 \mathrm{ng}$ DNA. The left panel shows the results for the unmethylated primer set (U) and the right panel for the methylated primer set (M). (b) MS-MLPA on serial dilutions of MCF10A breast epithelial cell line DNA. Results for ME001-C1 kit including RARB probe (193 nt) and two RASSF1 probes (328 and $382 \mathrm{nt}$ ). Amount of input DNA is indicated. Capillary electrophoresis results for digested samples. MS-MLPA is feasible down to a minimum of $10 \mathrm{ng}$ DNA. (c) QM-MSP for RARB on serial dilutions of MCF10A breast epithelial cell line DNA. Quantitative PCR methylation curves are shown. From left to right: 100, 10 and $1 \mathrm{ng}$ of input DNA for NaBi treatment. The distances between the three curves are not equal. Probably, more DNA is lost during column purification after $\mathrm{NaBi}$ treatment and amplification in multiplex PCR is less efficient for the higher DNA concentration. 


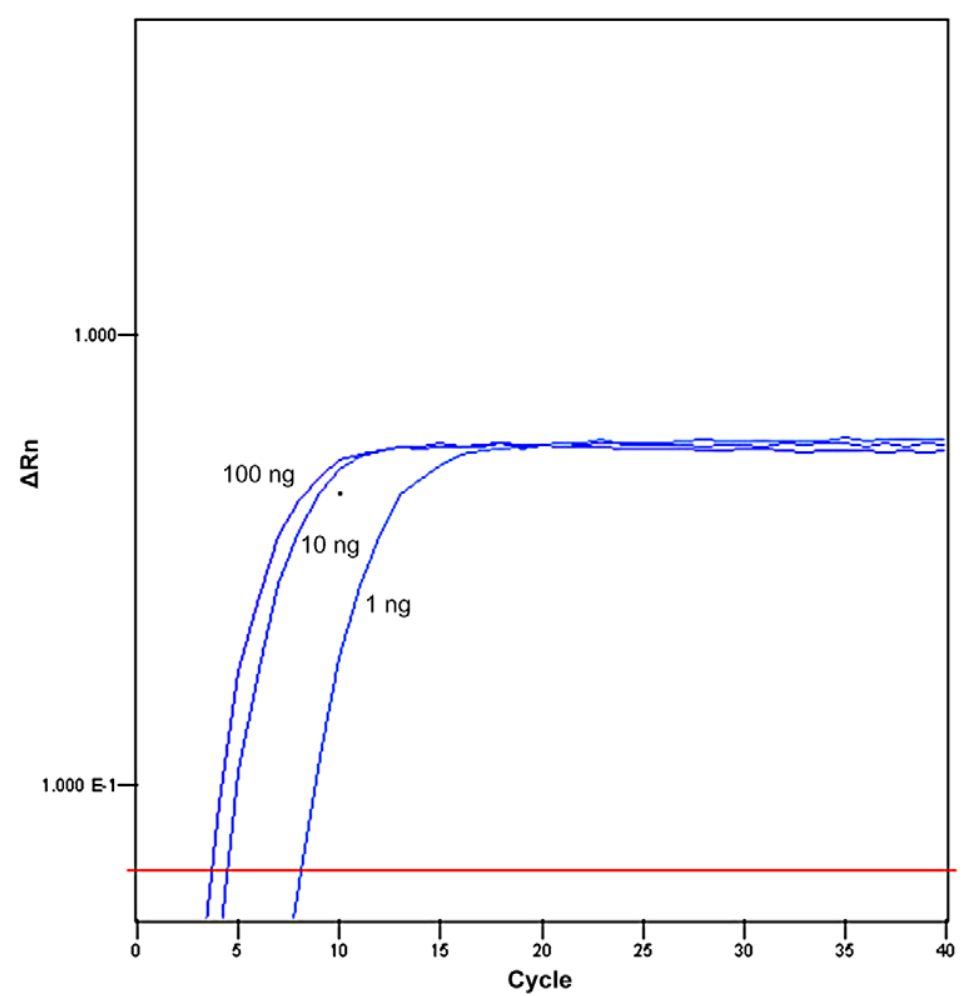

(c)

Fig. 3. (Continued.) (Colors are visible in the online version of the article; http://dx.doi.org/10.3233/ACP-CLO-2010-0542.)

QM-MSP appeared feasible in ten fold lower concentrations of DNA compared to non-quantitative MSP and MS-MLPA for RARB. Therefore, although they are both fast and simple procedures, MSP and MS-MLPA lack the sensitivity needed for the detection of methylation in the small amounts of DNA often present in biological fluids. Although MSP in biological fluids with scarce DNA has been reported, often a substantial part of the samples failed, or the amount of DNA recovered allowed only one gene to be analyzed [11].

Further, in larger amounts of DNA, MSP did not pick up significant methylation dosages as found with the quantitative assays MS-MLPA and QM-MSP reported here. In contrast to our findings, Jeuken et al. [9] demonstrated a good concordance between MSMLPA and (slightly modified) MSP for MGMT, when comparing them non-quantitatively using a $25 \%$ cutoff. In their study, percentages from four MS-MLPA probes for $M G M T$, a well-established predictive biomarker [25] were averaged, which together with the binary approach may account for the better accordance found. We did not analyze MGMT methylation in our study.
In this study, we chose the inter-assay concordance as a read-out. A gold standard was not available, since no technique can be regarded as universally superior [13]. Sequencing of sodium bisulfite treated DNA is often used as a gold standard, but this method also depends on the efficiency of $\mathrm{NaBi}$ conversion. Moreover, for the present study sequencing of sodium bisulfite treated DNA would anyhow not be the optimal reference since it would inherently incline more to the other $\mathrm{NaBi}$-based methods than to the restriction enzyme based assays. Finally direct sequencing of sodiumbisulfite treated DNA extracted from patient samples is very sensitive to contamination by wildtype cells as signals of methylated and unmethylated nucleotides are collected in the same sequence read. Lower levels of methylation are therefore likely to remain obscured in heterogeneous tumor cells populations mixed with stroma. Only after subcloning of a large number of individual DNA fragments followed by sequencing, quantitative methylation frequencies can be obtained. Therefore at present this technique is not amendable to routine diagnostic and clinical methylation analysis and not further addressed here. 
We conclude that despite the difference in molecular basis, the quantitative methylation assays QM-MSP and MS-MLPA show strong correlations when analyzing the same $\mathrm{CpG}$ islands. In contrast, there was a poor concordance between either of these quantitative techniques with non-quantitative MSP, which questions the validity of MSP for accurate methylation detection in clinical samples. Therefore, QM-MSP is the method of choice for biological samples with low amounts of DNA, such as biological fluids. MS-MLPA is a good and simple alternative when DNA quantities are not limiting.

\section{Acknowledgements}

We want to thank Folkert Morsink for his help with the figures and Vicky the Boer for her valuable contribution. This work was supported by the Dutch Cancer Society (UU 2007-3977 and UU 2008-4217), the "Integraal Kankercentrum Midden-Nederland (IKMN)", the "Ata Visser Stichting", the American Women's Club of The Hague, a Sister's Hope/Pink Ribbon and ZonWM (AGIKO stipend).

\section{References}

[1] E. Capel, J.F. Flejou and R. Hamelin, Assessment of MLH1 promoter methylation in relation to gene expression requires specific analysis, Oncogene 26 (2007), 7596-7600.

[2] J. Coffa, M.A. van de Wiel, B. Diosdado, B. Carvalho, J. Schouten and G.A. Meijer, MLPAnalyzer: data analysis tool for reliable automated normalization of MLPA fragment data, Cell. Oncol. 30 (2008), 323-335.

[3] M.J. Fackler, K. Malone, Z. Zhang, E. Schilling, E. GarrettMayer, T. Swift-Scanlan et al., Quantitative multiplex methylation-specific PCR analysis doubles detection of tumor cells in breast ductal fluid, Clin. Cancer Res. 12 (2006), 33063310.

[4] M.J. Fackler, M. McVeigh, J. Mehrotra, M.A. Blum, J. Lange, A. Lapides et al., Quantitative multiplex methylation-specific PCR assay for the detection of promoter hypermethylation in multiple genes in breast cancer, Cancer Res. 64 (2004), 4442 4452.

[5] E.H. Gort, K.P.M. Suijkerbuijk, S.M. Roothaan, V. Raman, M. Vooijs, E. van der Wall et al., Methylation of the TWIST1 promoter, TWIST1 mRNA levels, and immunohistochemical expression of TWIST1 in breast cancer, Cancer Epidemiol. Biomarkers Prev. 17 (2008), 3325-3330.

[6] J.G. Herman, J.R. Graff, S. Myohanen, B.D. Nelkin and S.B. Baylin, Methylation-specific PCR: A novel PCR assay for methylation status of CpG islands, PNAS 93 (1996), 98219826.
[7] M.O. Hoque, Q. Feng, P. Toure, A. Dem, C.W. Critchlow, S.E. Hawes et al., Detection of aberrant methylation of four genes in plasma DNA for the detection of breast cancer, J. Clin. Oncol. 24 (2006), 4262-4269.

[8] G.J. Jenkins, G.L. Williams, J. Beynon, Z. Ye, J.N. Baxter and J.M. Parry, Restriction enzymes in the analysis of genetic alterations responsible for cancer progression, $B r . J$. Surg. 89 (2002), 8-20.

[9] J.W.M. Jeuken, S.J.B. Cornelissen, M. Vriezen, M.M.G. Dekkers, A. Errami, A. Sijben et al., MS-MLPA: an attractive alternative laboratory assay for robust, reliable, and semiquantitative detection of MGMT promoter hypermethylation in gliomas, Lab. Invest. 87 (2007), 1055-1065.

[10] P.A. Jones and S.B. Baylin, The fundamental role of epigenetic events in cancer, Nat. Rev. Genet. 3 (2002), 415-428.

[11] R. Krassenstein, E. Sauter, E. Dulaimi, C. Battagli, H. Ehya, A. Klein-Szanto et al., Detection of breast cancer in nipple aspirate fluid by $\mathrm{CpG}$ island hypermethylation, Clin. Cancer Res. 10 (2004), 28-32.

[12] L.S. Kristensen and L.L. Hansen, PCR-based methods for detecting single-locus DNA methylation biomarkers in cancer diagnostics, prognostics, and response to treatment, Clin. Chem. 55 (2009), 1471-1483.

[13] P.W. Laird, The power and the promise of DNA methylation markers, Nat. Rev. Cancer 3 (2003), 253-266.

[14] C.M. Lewis, L.R. Cler, D.W. Bu, S. Zochbauer-Muller, S. Milchgrub, E.Z. Naftalis et al., Promoter hypermethylation in benign breast epithelium in relation to predicted breast cancer risk, Clin. Cancer Res. 11 (2005), 166-172.

[15] Z.J. Liu and M. Maekawa, Polymerase chain reaction-based methods of DNA methylation analysis, Anal. Biochem. 317 (2003), 259-265

[16] C.B. Moelans, R.A. de Weger, M.T.M. van Blokland, C. Ezendam, S. Elshof, M.G.J. Tilanus et al., HER-2/neu amplification testing in breast cancer by multiplex ligation-dependent probe amplification in comparison with immunohistochemistry and in situ hybridization, Cell. Oncol. 31(1) (2009), 1-10.

[17] A.O.H. Nygren, N. Ameziane, H.M.B. Duarte, R.N.C.P. Vijzelaar, Q. Waisfisz, C.J. Hess et al., Methylation-Specific MLPA (MS-MLPA): simultaneous detection of CpG methylation and copy number changes of up to 40 sequences, Nucl. Acids Res. 33 (2005), e 128.

[18] L. Pasquali, A. Bedeir, S. Ringquist, A. Styche, R. Bhargava and G. Trucco, Quantification of CpG island methylation in progressive breast lesions from normal to invasive carcinoma, Cancer Lett. 257 (2007), 136-144.

[19] G. Sharma, S. Mirza, Y.H. Yang, R. Parshad, P. Hazrah, S. Datta Gupta et al., Prognostic relevance of promoter hypermethylation of multiple genes in breast cancer patients, Cell. Oncol. 31 (2009), 487-500.

[20] K.P. Suijkerbuijk, M.J. Fackler, S. Sukumar, C.H. van Gils, T. van Laar, E. van der Wall et al., Methylation is less abundant in BRCA1-associated compared with sporadic breast cancer, Ann. Oncol. 19 (2008), 1870-1874.

[21] K.P.M. Suijkerbuijk, P.J. van Diest and E. van der Wall, Improving early breast cancer detection: focus on methylation, Ann. Oncol. (2010); doi: 10.1093/annonc/mdg305. [Epub June 29 2010.]

[22] K.P.M. Suijkerbuijk, E. van der Wall and P.J. van Diest, Oxytocin: bringing magic into nipple aspiration, Ann. Oncol. 18 (2007), 1743-1744. 
[23] T. Swift-Scanlan, A. Blackford, P. Argani, S. Sukumar and M.J. Fackler, Two-color quantitative multiplex methylationspecific PCR, Biotechniques 40 (2006), 210-219.

[24] P.J. van Diest, No consent should be needed for using leftover body material for scientific purposes, BMJ 325 (2002), 648651.

[25] M. Weller, R. Stupp, G. Reifenberger, A.A. Brandes, M.J. van den Bent, W. Wick et al., MGMT promoter methylation in ma- lignant gliomas: ready for personalized medicine?, Nat. Rev. Neurol. 6 (2010), 39-51.

[26] P.S. Yan, C. Venkataramu, A. Ibrahim, J.C. Liu, R.Z. Shen and N.M. Diaz et al., Mapping geographic zones of cancer risk with epigenetic biomarkers in normal breast tissue, Clin. Cancer Res. 12 (2006), 6626-6636. 


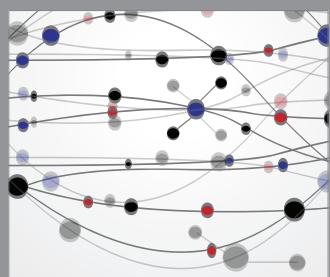

The Scientific World Journal
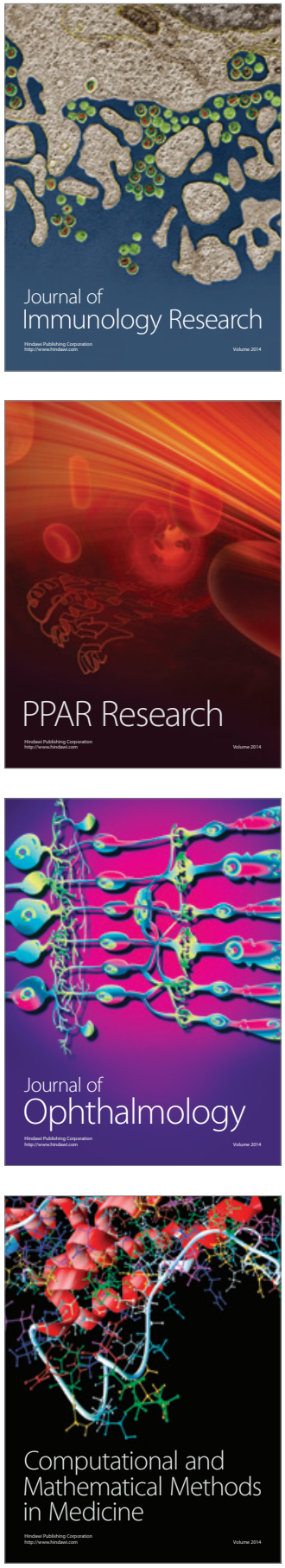

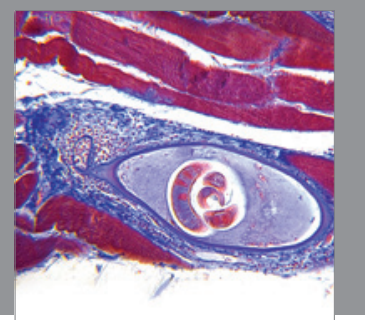

Gastroenterology

Research and Practice
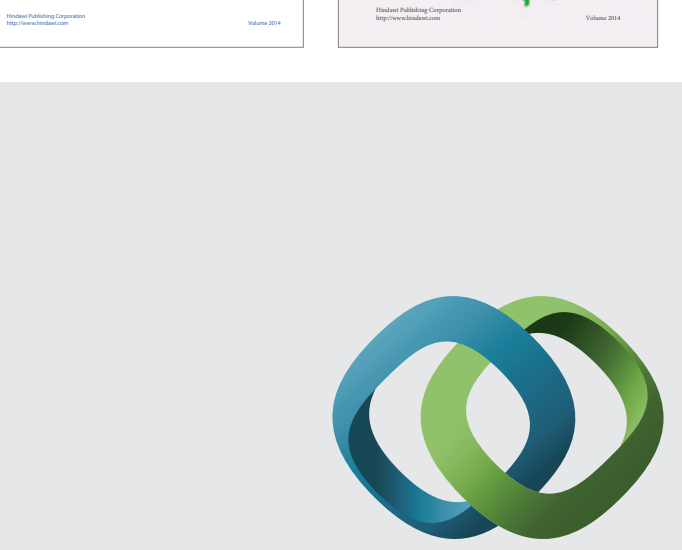

\section{Hindawi}

Submit your manuscripts at

http://www.hindawi.com
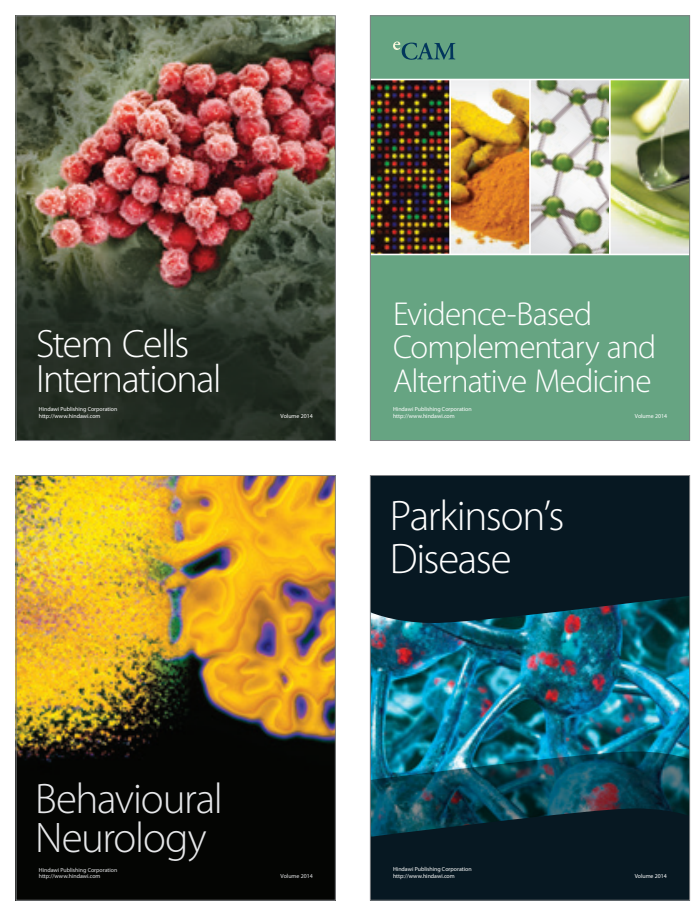

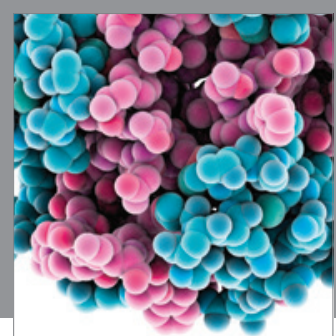

Journal of
Diabetes Research

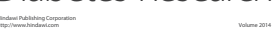

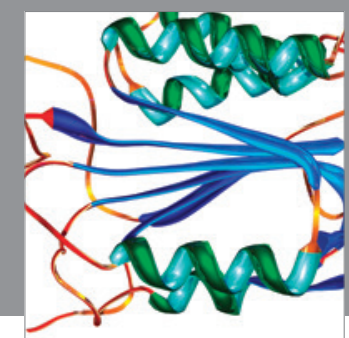

Disease Markers
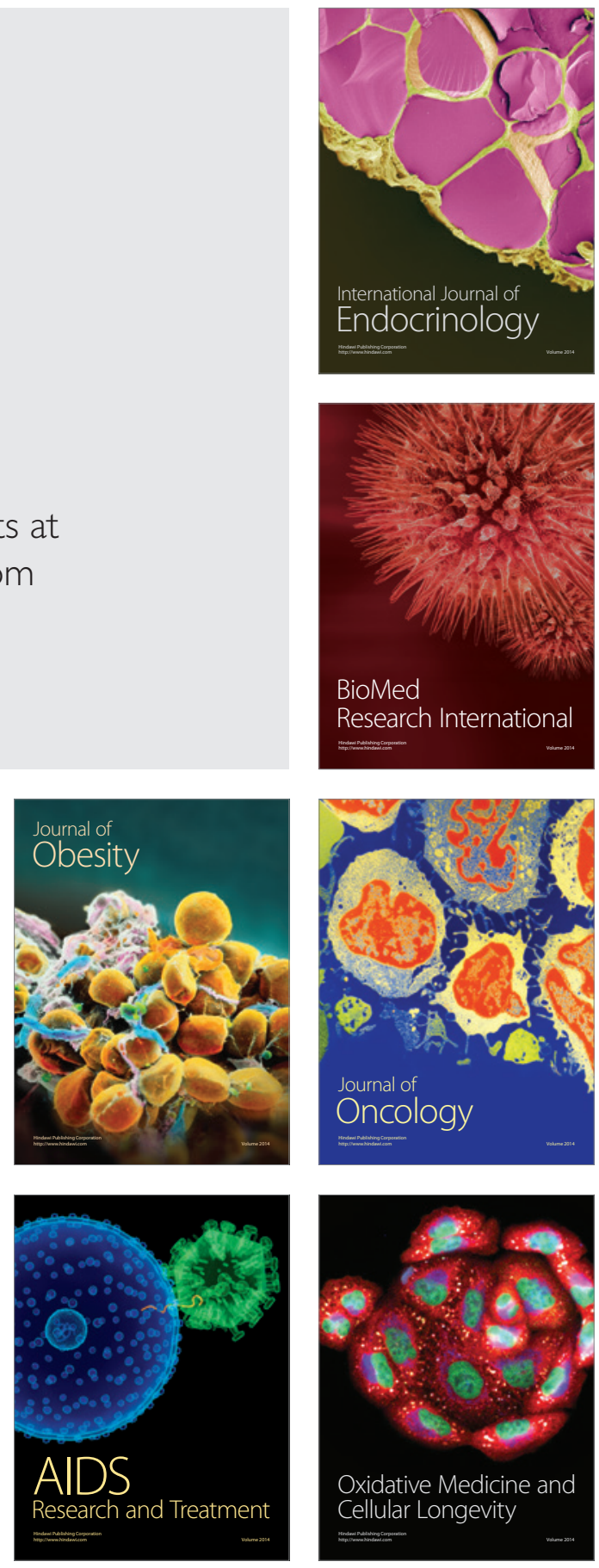